\title{
A!
}

This is an electronic reprint of the original article.

This reprint may differ from the original in pagination and typographic detail.

Fazio, Rosario; Hekking, F.W.; Pekola, Jukka

\section{Measurement of coherent charge transfer in an adiabatic Cooper-pair pump}

Published in:

Physical Review B

DOI:

10.1103/PhysRevB.68.054510

Published: 07/08/2003

Document Version

Publisher's PDF, also known as Version of record

Please cite the original version:

Fazio, R., Hekking, F. W., \& Pekola, J. (2003). Measurement of coherent charge transfer in an adiabatic Cooperpair pump. Physical Review B, 68(5), 1-11. [054510]. https://doi.org/10.1103/PhysRevB.68.054510

This material is protected by copyright and other intellectual property rights, and duplication or sale of all or part of any of the repository collections is not permitted, except that material may be duplicated by you for your research use or educational purposes in electronic or print form. You must obtain permission for any other use. Electronic or print copies may not be offered, whether for sale or otherwise to anyone who is not an authorised user. 


\title{
Measurement of coherent charge transfer in an adiabatic Cooper-pair pump
}

\author{
Rosario Fazio \\ NEST-INFM \& Scuola Normale Superiore, 56126 Pisa, Italy \\ F. W. J. Hekking \\ Laboratoire de Physique et Modélisation des Milieux Condensés Magistère-CNRS, Boîte Postale 166, 38042 Grenoble cedex 9, France \\ J. P. Pekola \\ Low Temperature Laboratory, Helsinki University of Technology, P.O. Box 2200, FIN-02015 HUT Espoo, Finland
}

(Received 19 February 2003; published 7 August 2003)

\begin{abstract}
We study adiabatic charge transfer in a superconducting Cooper-pair pump, focusing on the influence of current measurement on coherence. We investigate the limit where the Josephson coupling energy $E_{J}$ between the various parts of the system is small compared to the Coulomb charging energy $E_{C}$. In this case, the charge transferred in a pumping cycle $Q_{P} \sim 2 e$, the charge of one Cooper pair: The main contribution is due to incoherent Cooper-pair tunneling. We are particularly interested in the quantum correction to $Q_{P}$, which is due to coherent tunneling of pairs across the pump and which depends on the superconducting phase difference $\varphi_{0}$ between the electrodes; $1-Q_{P} /(2 e) \sim\left(E_{J} / E_{C}\right) \cos \varphi_{0}$. A measurement of $Q_{P}$ tends to destroy the phase coherence. We first study an arbitrary measuring circuit and then specific examples and show that coherent Cooper-pair transfer can, in principle, be detected using an inductively shunted ammeter.
\end{abstract}

DOI: $10.1103 /$ PhysRevB.68.054510

PACS number(s): 74.50. $+\mathrm{r}, 74.78 . \mathrm{Na}, 73.23 . \mathrm{Hk}$

\section{INTRODUCTION}

It is well known that electrons can be transferred through a mesoscopic device by means of adiabatic changes of system parameters, e.g., externally applied electric or magnetic fields. Since the original work of Thouless, ${ }^{1}$ several theoretical proposals concerning possible physical realizations of this phenomenon, usually referred to as parametric pumping, have been put forward. ${ }^{2}$ Depending on the physical mechanism employed, these proposals can be divided into two classes.

In open systems, i.e., systems consisting of several conducting parts, connected to each other by highly transmissive barriers, parametric pumping can be achieved through a periodic modulation of phase of the scattering matrix associated to the device. ${ }^{3}$ In these proposals, electronic phase coherence plays a fundamental role and charge is transferred coherently through the entire system. The amount of charge that is transferred per period of the modulation is, in general, a fraction of the electronic charge $e$, which depends on the modulation path. In open devices, electron-electron interactions are weak and lead to (small) dephasing corrections to the noninteracting result for charge transfer.

In the opposite limit of closed systems, i.e., several metallic islands connected to each other by ultrasmall tunnel junctions, parametric pumping of charge is achieved by periodic modulation of the so-called Coulomb blockade. ${ }^{4}$ In these proposals, the presence of strong Coulomb repulsion between electrons is essential. It leads to the quantization of charge on the islands in units of $e$. A periodic modulation of externally applied gate voltages leads to a periodic lifting of Coulomb blockade, which enables the transfer of exactly one electron per period through the device. The presence of phase coherence in this limit only leads to small corrections to the classical result, through coherent higher-order chargetransfer processes known as (in)elastic cotunneling. ${ }^{5}$

Experimental evidence for parametric charge pumping in normal metallic systems has been found in both limits of open $^{6}$ and closed ${ }^{7,8}$ systems.

The present paper is devoted to parametric pumping in a superconducting system. ${ }^{9}$ It consists of superconducting islands, connected to each other by small tunnel junctions, and it is operated in the Coulomb blockade regime. From this point of view the system is closed, and charge transfer is mainly classical, i.e., quantized in units of Cooper-pair charge $2 e$ per period of the modulation. On the other hand, phase is defined naturally in a superconducting system, and due to the Josephson coupling between the various parts of the system phase coherence tends to be maintained throughout the device. In this sense, a superconducting pump behaves as an open system, and the total charge transfer will be characterized by significant phase-coherent corrections to the classical result. ${ }^{10}$

We will be particularly interested in the influence of the measurement apparatus of the amount of transferred charge on the phase-coherent properties of a superconducting charge pump. Measurement on a quantum coherent system is achieved by connecting it to the environment provided by the measuring device. The environment in turn can be modeled as a collection of harmonic oscillators. Depending on the environment, the quantum coherence is typically lost in time which we can call the dephasing or the decoherence time once properly defined. We focus here on quantum coherent systems formed of Josephson tunnel junctions and their electromagnetic environment. These systems, ${ }^{11-14}$ or we might already call some of them devices to perform quantum logic operations (see review in Ref. 15), hold great promise in quantum computing because of their potential in scalability. 

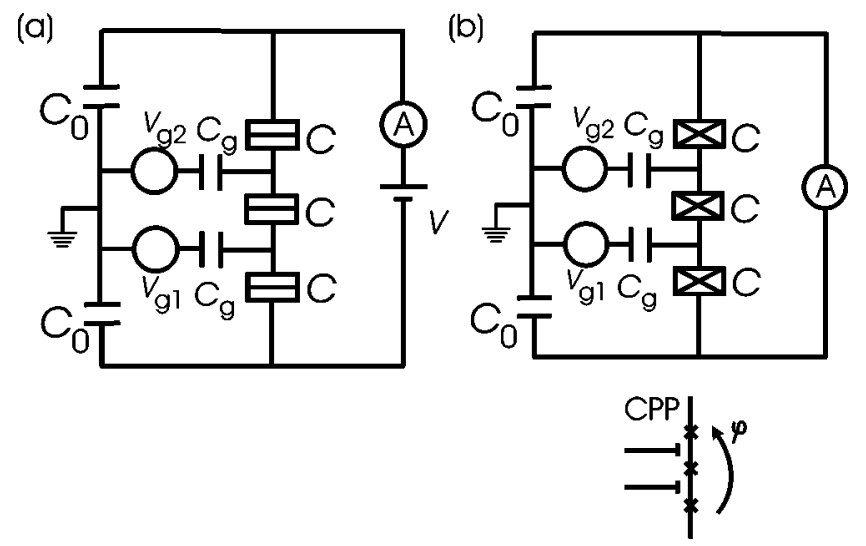

FIG. 1. Double island (a) electron and (b) Cooper-pair pump. A symmetric pump consists of junctions with capacitance $C$ and Josephson coupling energy $E_{J}$. Gate capacitance is $C_{g}$ for both islands and the gate voltages are $V_{g 1}$ and $V_{g 2}$ for the first and the second island, respectively. Electron pump can be operated with either zero or nonzero bias voltage $V$ across it; Cooper-pair pump has zero-bias voltage but it can be phase biased by $\varphi$. In (b), we also show the shorthand symbol used in the later figures for a CPP with phase $\varphi$ across it.

In a superconducting system, the phase difference across a junction follows the well-known ac and dc Josephson relations. The theory of phase fluctuations and environment is well established for electrical circuits including small tunnel junctions. ${ }^{16-18}$ What we do here specifically is the analysis of the back action of the measurement of electrical current on a circuit consisting of small Josephson junctions. In particular, we show that unlike in a standard dissipative ammeter, the phase diffusion is limited in a measurement performed by an inductively shunted measuring circuit. Measurement of tiny currents provides a readout of Josephson-junction quantum bits, such as the one in the so-called "quantronium" experiment ${ }^{14}$ or, in general, when reading out the persistent current in a flux quantum bit [an rf Superconducting quantum interference device (SQUID) loop]. ${ }^{12,13}$ As a specific example, we focus on a measurement of current in a double island adiabatic Cooper-pair pump (CPP), but our conclusions concerning the effect of an inductance limited phase diffusion measurement can easily be generalized to other Josephson-junction circuits with straightforward modifications, which account for the different topology of the circuit to be measured.

\section{THE MODEL}

An electron pump is a reversible device which provides quantized transport of electrical charge upon cyclic operation of gates connected to it. The simplest variant is perhaps a double island pump [see Fig. 1(a)] in which tiny metallic (nonsuperconducting) or semiconducting grains are tunnel coupled to each other and to the surrounding electrodes, and capacitively coupled to two gates. ${ }^{7,8}$ The islands are so small that, due to their tiny capacitance, equilibrium charge configurations on them are determined solely by electrostatics on the single-electron level characterized by the scale of charging energy $E_{C}$. Applying $\pi / 2$ phase-shifted harmonic voltages to the two gates at a frequency $f$, which is so low that it allows the system to follow the ground-state configuration of charging energy at each phase of operation, current through the pump equals $I_{P}=e f$, based on transport of one electron per cycle, on a precision level of a few percent. A more accurate but more complicated device can be built by increasing the number of islands and adjacent gates in the pump this approach has been taken to meet with requirements in metrology. ${ }^{19}$

If all the electrodes and islands are superconducting [see Fig. 1(b)], charge can be transported not only as singleelectrons, or rather quasiparticles with single electron charge in this case, but also as Cooper-pairs. ${ }^{9,10}$ Ideally, in the absence of any bias voltage and at low temperatures $T$ such that $k_{B} T \ll E_{C} \ll \Delta$, where $\Delta$ is the superconducting gap and $E_{C}$ $=2 e^{2} / C$ ( $C$ is the junction capacitance), the system is in the Coulomb blockade regime, and only Cooper pairs are transferred. In this regime, the device can be referred to as a CPP, described by the Hamiltonian

$$
\hat{H}=\hat{H}_{C}+\hat{H}_{J}
$$

where

$$
\hat{H}_{C}=\frac{2}{3} E_{C}\left[\left(\hat{n}_{1}-n_{x 1}\right)^{2}+\left(\hat{n}_{2}-n_{x 2}\right)^{2}+\left(\hat{n}_{1}-n_{x 1}\right)\left(\hat{n}_{2}-n_{x 2}\right)\right]
$$

is the charging energy of the islands, coupled capacitively to each other as well as to two gates 1 and 2. The operator $\hat{n}_{1(2)}$ denotes the number of excess Cooper pairs on island $1(2)$ and $n_{x 1(x 2)}$ is the gate charge (in units of $2 e$ ) which can be tuned by varying the gate voltage $V_{g 1(g 2)}$. The Josephson contribution is given by

$$
\begin{aligned}
\hat{H}_{J}= & -E_{J}\left[\cos \left(\varphi / 3+\hat{\phi}_{1}\right)+\cos \left(\varphi / 3+\hat{\phi}_{2}-\hat{\phi}_{1}\right)\right. \\
& \left.+\cos \left(\varphi / 3-\hat{\phi}_{2}\right)\right] .
\end{aligned}
$$

Here, $E_{J}$ is the Josephson coupling energy of each junction (assumed equal for the three junctions). Throughout this paper, we will work in the limit $E_{J} \ll E_{C}$ where charging effects dominate. The phase operator $\hat{\phi}_{1(2)}$ is conjugate to the number operator $\hat{n}_{1(2)}$. The phase bias $\varphi$ is the phase difference across the entire pump. As long as one can ignore any dynamical environment, $\varphi$ can be considered as a classical variable that can be set to a constant offset value $\varphi=\varphi_{0}$, for instance, by applying a magnetic flux through the circuit of Fig. 1(b).

We are interested in the charge transferred through the device upon a periodic modulation of the applied gate charges $n_{x 1}(t)$ and $n_{x 2}(t)$. Specifically, we assume the gate modulation to be adiabatically slow, with a frequency $f \ll E_{J}^{2} / \hbar E_{C}$. Under this condition, the system remains in the ground state throughout the modulation if the temperature is low, $k_{B} T \ll E_{J}$. During one period of the modulation, the two-component vector $\vec{n}_{x}=\left(n_{x 1}, n_{x 2}\right)$ describes a closed path in the corresponding two-dimensional plane, see, e.g., Fig. 2. It can be shown ${ }^{10}$ that the transferred charge in the ground 


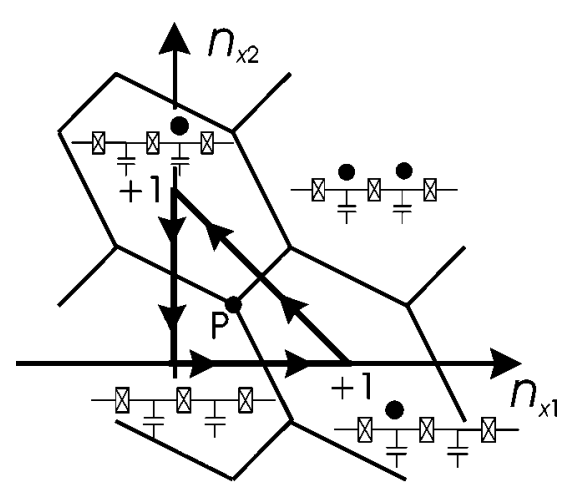

FIG. 2. Triangular gating sequence in the $n_{x 1}, n_{x 2}$ plane for the pumping cycle as discussed in the text. Hexagons correspond to regions in which the-schematically indicated-classical charge configurations are stable.

state during one period of the modulation is given by a contour integral along the closed path followed by $\vec{n}_{x}$,

$$
Q_{P}=2 \hbar \operatorname{Im}\left[\sum_{n \neq 0} \oint \frac{(\hat{I})_{0 n}}{E_{0}-E_{n}}\left\langle n \mid \partial_{n_{x}} 0\right\rangle \cdot d \vec{n}_{x}\right] .
$$

Here, we introduced the instantaneous eigenstates $|m\rangle$ and energies $E_{m}$ of the corresponding time-dependent Hamiltonian (1), such that $\hat{H}(t)|m(t)\rangle=E_{m}(t)|m(t)\rangle$. The matrix element $(\hat{I})_{0 n}$ corresponds to the current operator of one of the junctions. For the leftmost junction, for instance, we have

$$
\hat{I} \equiv \hat{I}_{l}=I_{J} \sin \left(\hat{\phi}_{1}+\varphi / 3\right),
$$

where $I_{J}=2 e E_{J} / \hbar$ is the Josephson critical current. The matrix element is taken between the instantaneous ground state $|0\rangle$ and the instantaneous excited state $|n\rangle$. An outline of the derivation of Eq. (4) is given in Appendix A.

In the limit $E_{J} / E_{C} \rightarrow 0$, to leading-order tunneling events involving more than one junction can be ignored. As a result, a periodic gate modulation will lead to a transfer of a charge $Q_{P}=2 e$ per cycle through the pump, independent of the bias phase $\varphi_{0}$, see Appendix . This is the limit of incoherent Cooper-pair tunneling. Experimentally, incoherent Cooperpair tunneling is best realized by a sufficiently dissipative environment which completely randomizes $\varphi_{0}$, e.g., by fabricating on-chip resistors near the pump, with resistances of the order or larger than the resistance quantum for Cooperpairs: $R_{K} / 4=h /(2 e)^{2} \simeq 6.45 \mathrm{k} \Omega .^{20}$

Deviations from the classical result are related to higherorder tunneling processes. In general, pumps in the superconducting state are less accurate than their normal-state counterparts and one fundamental reason is the coherence of the superconducting wave function. In this paper, we are interested in the regime where coherent Cooper-pair tunneling leads to an important correction to the incoherent charge transfer. It provides an interesting, still unobserved quantummechanical interference correction to the pumped charge (or pumped current in continuous cycling): ${ }^{10}$

$$
Q_{P} / 2 e \simeq 1-9\left(E_{J} / E_{C}\right) \cos \varphi_{0}
$$

(a)

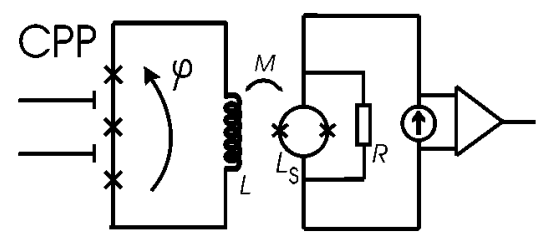

(b)

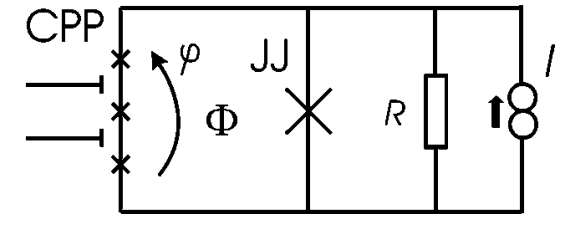

FIG. 3. Two specific examples of inductive measuring circuits for current. In (a) the SQUID ammeter is inductively coupled to the measured circuit. In (b) current is measured by determining the escape probability from the zero voltage state of a Josephson junction. The inductance is provided by the measuring junction. The phase $\varphi$ can be adjusted by, e.g., applying a flux $\Phi$ through the loop of the CPP and the measuring circuit.

This result is obtained for a triangular gating, as shown in Fig. 2; some details of the derivation are given in Appendix . Although the result is perturbative in $E_{J} / E_{C}$, the coherent interference term may be appreciable. For example, for $E_{J} / E_{C}=0.1$, still rather well within the perturbative regime, the variation of the pumped current $I_{P} \equiv Q_{P} f$ is 1.8 times larger than the magnitude of the incoherent current $I_{\text {inc }}$ $\equiv 2 e f$. The coherent contribution to the current can be tuned by adjusting $\varphi_{0}$. By assuming a realistic operating frequency of $f=100 \mathrm{MHz}, 2$ ef equals $32 \mathrm{pA}$, and this would produce variation in the interference term by $58 \mathrm{pA}$. Detecting this phase coherent current would not only be fundamentally interesting but it would also allow one to measure dephasing in a superconducting multijunction circuit coupled to a measuring device.

We finally note that, in addition to the current $I_{P}=Q_{P} f$ discussed so far, a direct Josephson current $I_{\text {jos }}$ exists in response to the phase bias $\varphi_{0}$. The Josephson current is maximum, $\sim I_{J}$, at the triple point $P$ in Fig. 2, where the three charge configurations $|0,0\rangle,|1,0\rangle$, and $|0,1\rangle$ are degenerate. The gating sequence of interest here is away from $P$ : at any given point along the cycle at most two out of the three aforementioned relevant charge configurations are degenerate. At such a degeneracy, the Josephson current is smaller, $\sim I_{J}\left(E_{J} / E_{C}\right)$. The width of the degeneracy is finite, $\propto E_{J} / E_{C}$. Hence, only a fraction of the total cycle contributes to the Josephson current and $I_{\text {jos }} \propto I_{J}\left(E_{J} / E_{C}\right)^{2} \sin \varphi_{0}$. Thus, $I_{\text {jos }}$ can be distinguished from $I_{P}$, as it depends differently on $\varphi_{0}$ and is independent of the frequency $f$.

\section{COUPLING TO A MEASUREMENT CIRCUIT}

We propose two strategies to measure the pumped charge as illustrated in Fig. 3. The first variant, in Fig. 3(a), is a generic inductively coupled measurement by a dc SQUID (input coil $L$, mutual inductance $M$, shunt resistance $R$ ). We call this circuit $\mathbf{A}$ in the following. The second circuit, in Fig. 
(a)

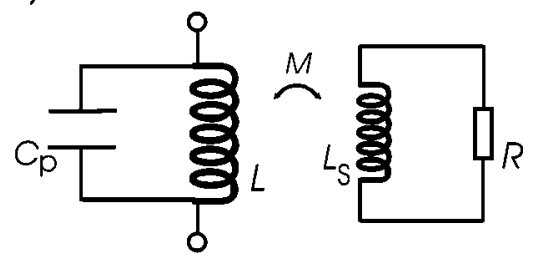

(b)

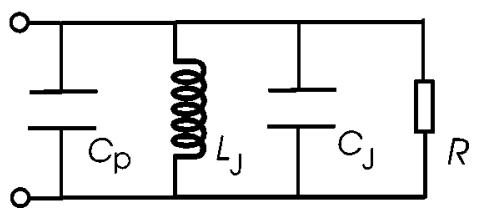

FIG. 4. Circuit models of the two measuring devices: in (a) we model the SQUID by an inductor with inductance $L_{s}$ and in (b) the Josephson junction is represented by a parallel $L R C$ circuit.

3(b), represents a directly coupled measurement of current by a Josephson junction. In this configuration, changes in current of the measured circuit are detected as changes of lifetime of a metastable zero voltage (supercurrent) state of the measuring current biased junction. ${ }^{14,21}$ This measurement circuit will be called $\mathbf{B}$ in what follows. The measuring junction is biased with a current $I$; its capacitance $C_{J}$ is the capacitance due to its typically planar geometry. When biased near the critical current, $I \leqslant I_{c}$, the junction can be characterized by the Josephson inductance $L_{J}=\sqrt{2}\left(\hbar / 2 e I_{c}\right)(1$ $\left.-I / I_{c}\right)^{-1 / 2}$. This relation conforms with the identity between the plasma frequency $\omega_{p} / 2 \pi$ and the circuit parameters of the junction: $\omega_{p}=\left(L_{J} C_{J}\right)^{-1 / 2}$.

The equivalent circuits corresponding to $\mathbf{A}$ and $\mathbf{B}$ are shown in Figs. 4(a) and 4(b), respectively. In (a) we assume the SQUID to be a pure inductor of inductance $L_{s}$. In both (a) and (b) $C_{p}$ represents the total capacitance across the pump circuit, including half of the ground capacitance $C_{0}$ of the (symmetric) pump loop. We assume that the gate capacitances of the pump are small and can be neglected. In (b) the current biased measuring junction is replaced by its resistively and capacitively shunted junction (RCSJ) equivalent.

We do not consider in detail the exact electromagnetic environment. But, as will be obvious, the value of the resistance in shunting the SQUID in $\mathbf{A}$ or the junction in $\mathbf{B}$ does not influence our general conclusion. Furthermore, the $L C$-type lines in the environment, not included in the present discussion, further help to decouple the dissipative environment from the measured circuit. In $\mathbf{A}$ we can assume that the parallel amplifier impedance and in $\mathbf{B}$ the dissipative (real) part of the environment are included in $R$.

A few words on the current resolution $\delta I$ of the two circuits are in order. In an optimum measurement by circuit $\mathbf{A}$, $\delta I$ is quantum limited to $\delta I / \sqrt{\Delta f} \sim \sqrt{4 \pi \hbar / L}$, where $\Delta f$ is the bandwidth of the measurement. This holds for perfect coupling to the SQUID. Inserting $L=100 \mathrm{nH}$, we obtain $\delta I / \sqrt{\Delta f} \sim 120 \mathrm{fA}$ and for the bandwidth $\Delta f=10 \mathrm{kHz}$, we have $\delta I \sim 12 \mathrm{pA}$. For faster measurement, one needs higher input inductance: to achieve 10-pA resolution at $1 \mathrm{MHz}, L$ should exceed $10 \mu \mathrm{H}$, which starts to influence the pump operation itself, as will be discussed below. The current resolution of circuit $\mathbf{B}$ depends on optimizing its performance through the measuring junction characteristics. The ultimate limit of resolution can be reached when the junction is in the macroscopic quantum tunneling (MQT) limit, where escape to a finite voltage state is via tunneling through the washboard potential and thermal escape over the barrier is prohibited due to low temperature. Using a trapezoidal current pulse of height $I$ and duration $\Delta t$, we then have the probability $P=1-\exp (-\Gamma \Delta t)$ to escape from the zero voltage state, $\Gamma$ is the MQT escape rate. Since $\Gamma$ depends on the bias current $I$, we can use $P$ as a measure of the total current through the junction: ideally, this current is the sum of the bias current and the current to be measured, as suggested by Vion et al. ${ }^{14}$ whereby a figure of merit of the measurement is $\delta I=\delta P /(\partial P / \partial I)$ giving the smallest resolvable current. Here, $\delta P$ is the resolution in reading $P$, which for typical averaging is of the order of 0.01. By a straightforward analysis, we find out that there is a tradeoff of junction parameters in the sense that by reducing $\delta I$ (by increasing the ratio $C_{J} / I_{c}$ ), we lower at the same rate the crossover temperature $T_{0}$ from thermally activated escape to MQT. Using this, we can find an approximate answer $\partial P / \partial I \sim(\hbar / 2 e) /\left(k_{B} T_{0}\right)$. This, in turn, with $\delta P=0.01$, and by setting $T_{0}=30 \mathrm{mK}$, a realistic electron temperature in an experiment, yields $\delta I$ $\sim 10 \mathrm{pA}$. The bandwidth of this measurement depends on various technical parameters in the setup and we will not discuss it here. The comparison of the two measurements is limited by the fact that they present fundamentally different approaches. Circuit $\mathbf{A}$ aims at a continuous monitoring of the current at a certain bandwidth. Circuit $\mathbf{B}$ in turn tries to grab information of the CPP current in a single shot or by successive current pulses into the escape junction. Both of them can achieve the requested current resolution, but the suitability of the two depends on how they interfere with the measured CPP and whether one can initialize the CPP controllably. One of the advantages of circuit $\mathbf{B}$ is that the phase fluctuations are smaller owing to the smaller inductance of this detector.

In the presence of the measuring circuit, the bias phase difference $\varphi$ acquires its own dynamics. As a result, the phase will contain a fluctuating part, $\varphi(t)=\varphi_{0}+\phi(t)$, and this will, in general, modify the pumped charge. Before studying this modification in detail for the setups of Fig. 3, we analyze the time dependence of phase fluctuations induced by the measuring circuits. Fluctuation-dissipation theorem implies that the mean square of the fluctuations obeys the relation

$$
\left\langle\phi^{2}(t)\right\rangle=8 \int_{0}^{\infty} \frac{d \omega}{\omega} \frac{\operatorname{Re} Z(\omega)}{R_{K}} \operatorname{coth}\left(\frac{\hbar \omega}{2 k_{B} T}\right)[1-\cos (\omega t)] .
$$

In quantifying the phase fluctuations, we thus need to know the real (dissipative) part of the impedance seen by the measured circuit, $\operatorname{Re} Z(\omega)$, where $\omega$ is the (angular) frequency.

Circuit A. In this case, we obtain 


$$
\operatorname{Re} Z_{A}(\omega)=\frac{M^{2} R \omega^{2}}{R^{2}\left(1-L C_{p} \omega^{2}\right)^{2}+\omega^{2} L_{s}^{2}\left[1-(1-\gamma) L C_{p} \omega^{2}\right]^{2}},
$$

with $\gamma=M^{2} /\left(L_{s} L\right)$. For typical experimental values this represents a narrow resonance peak at $\omega \simeq \omega_{L C}$, where $\omega_{L C}$ $\equiv\left(L C_{p}\right)^{-1 / 2}$ is the natural $L C$ resonance (angular) frequency of the CPP circuit alone. We first study the limit $\gamma \ll 1$, i.e., weak coupling of the measuring circuit. The circuit can then behave in two different ways depending on whether the parameter $\alpha^{2} \equiv\left(\omega_{L C} L_{s} / R\right)^{2}$ is either $\ll 1$ or $\gg 1$. In the first limit, the resonant frequency is $\omega_{L C}$ and the width of the peak is $\delta \omega / \omega_{L C} \simeq(M / L)^{2} \sqrt{L / C_{p}} /(2 R)=\gamma \alpha / 2 \ll 1$. The product of the width and height of the resonance peak is $\operatorname{Re} Z(\omega)_{\max } \delta \omega \simeq \omega_{L C} \sqrt{L / C_{p}}$, independent of $R$. In the second limit, $\alpha^{2} \gg 1$, which corresponds to most experimental conditions, the measuring circuit decreases the effective inductance of the resonant circuit and the resonant frequency [maximum of $\operatorname{Re} Z_{A}(\omega)$ ] is shifted very slightly upwards to $\omega_{L C} / \sqrt{1-\gamma}$. The width of the peak is now $\delta \omega / \omega_{L C}$ $\simeq\left(M / L_{s}\right)^{2} R /\left(2 \sqrt{L / C_{p}}\right)=\gamma /(2 \alpha) \ll 1$. The product of the width and height is again $\operatorname{Re} Z(\omega)_{\max } \delta \omega \simeq \omega_{L C} \sqrt{L / C_{p}}$, independent of $R$.

From Eqs. (7) and (8), we see that in both limits the phase fluctuations do not diverge with time. Instead, we have initial oscillations which dephase and asymptotically the fluctuations tend to a constant expectation value. This behavior is not so unexpected since the measured circuit forms essentially a SQUID loop, where the average voltage over the junctions vanishes. The decay time of coherent phase oscillations turns out to be $t_{d e c, A}=L C_{p} R /\left(\gamma L_{s}\right)$ when $\alpha^{2} \ll 1$ and $t_{d e c, A}=L_{s} /(\gamma R)$ in the opposite limit. We obtain the following expressions for the mean square in the short- and longtime limits, respectively,

$$
\begin{aligned}
\left\langle\phi^{2}(t)\right\rangle \simeq & 4 \pi\left(\frac{\sqrt{L / C_{p}}}{R_{K}}\right) \operatorname{coth}\left(\frac{\hbar \omega_{L C}}{2 k_{B} T}\right) \\
& \times\left\{\begin{array}{l}
{\left[1-\cos \left(\omega_{L C} t\right)\right] \quad \text { if } t \ll t_{d e c, A}} \\
1 \quad \text { if } t \gg t_{d e c, A} .
\end{array}\right.
\end{aligned}
$$

In the opposite limit of strong coupling of the measuring circuit, $\gamma \rightarrow 1$, we essentially approach circuit $\mathbf{B}$ to be discussed below.

Circuit B. In this case, we have

$$
\operatorname{Re} Z_{B}(\omega)=\frac{L_{J}^{2} R \omega^{2}}{R^{2}\left(1-L_{J} C_{J}^{*} \omega^{2}\right)^{2}+\omega^{2} L_{J}^{2}}
$$

with $C_{J}^{*} \equiv C_{J}+C_{p}$. In this case, the low damping limit corresponds to $\delta \omega / \omega_{L C} \simeq \sqrt{L_{J} / C_{J}^{*}} / R \ll 1$, where $\omega_{L C}$ $\equiv\left(L_{J} C_{J}^{*}\right)^{-1 / 2}$ is again the $L C$ resonance (angular) frequency of the CPP circuit now with the measuring junction. In this limit, the maximum of the real impedance is $\operatorname{Re} Z_{B}(\omega)_{\max }$ $\simeq R$ and the product of height and width is $\operatorname{Re} Z_{B}(\omega)_{\max } \delta \omega \simeq \omega_{L C} \sqrt{L_{J} / C_{J}^{*}}$, again independent of $R$.
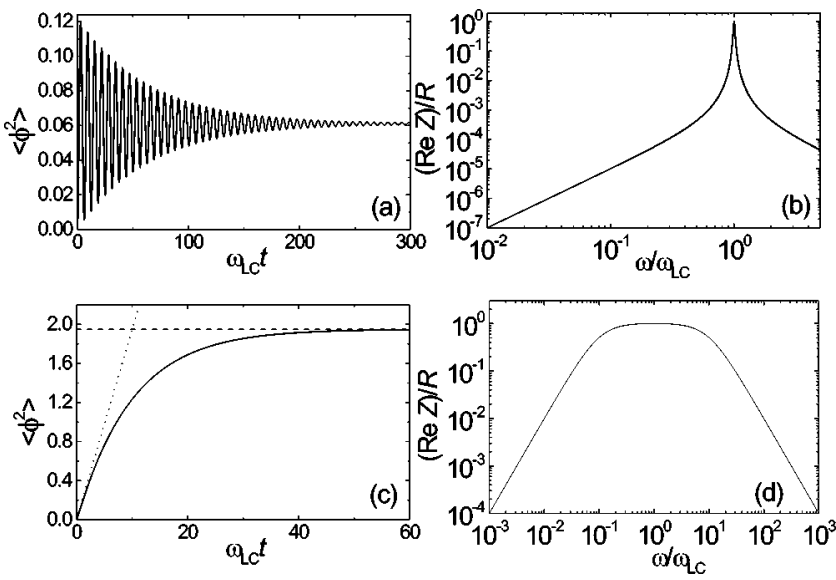

FIG. 5. (a) Numerically calculated $\left\langle\phi^{2}\right\rangle$ for circuit B. In (b) we show $\operatorname{Re} Z(\omega)$. The parameter values for these graphs are $L_{J}$ $=0.1 \mathrm{nH}, C_{J}^{*}=0.1 \mathrm{pF}, R=1 \mathrm{k} \Omega, T=50 \mathrm{mK}$, whereby $\delta \omega / \omega_{L C}$ $=0.03162$ and $\omega_{L C}=3.162 \times 10^{11} \mathrm{~s}^{-1}$. (c) and (d): demonstration of the influence of the overdamped inductive environment. For both graphs, we used the following parameters: $\delta \omega / \omega_{L C}=10$, $\hbar \omega_{L C} / k_{B} T=0.1, R=5 \Omega$. The predictions of Eqs. (12) and (13) are shown by the dotted and the dashed lines, respectively, and they intersect at $\omega_{L C} t=\delta \omega / \omega_{L C}$.

The coherence of oscillations in the underdamped case $\delta \omega / \omega_{L C} \ll 1$ dies in a time $t_{d e c, B}=2 R C_{J}^{*}$, and as a result, the asymptotics of the mean square for short and long times are given by

$$
\begin{aligned}
\left\langle\phi^{2}(t)\right\rangle \simeq & 4 \pi\left(\frac{\sqrt{L / C_{J}^{*}}}{R_{K}}\right) \operatorname{coth}\left(\frac{\hbar \omega_{L C}}{2 k_{B} T}\right) \\
& \times \begin{cases}{\left[1-\cos \left(\omega_{L C} t\right)\right] \quad \text { if } t \ll t_{d e c, B}} \\
1 & \text { if } t \gg t_{d e c, B} .\end{cases}
\end{aligned}
$$

It is interesting to see how the resonance behavior characteristic for circuits $\mathbf{A}$ and $\mathbf{B}$ transforms into an essentially diffusive one when the quality factor of the resonance in the dissipative $L C$ circuit gets lower. Let us consider circuit $\mathbf{B}$ as the example. In the overdamped case, $\delta \omega / \omega_{L C} \gg 1$, the phase fluctuations are diffusive when $t \ll t_{d e c}$. In particular, when $k_{B} T \gg \hbar \omega_{L C}$, we have

$$
\left\langle\phi^{2}(t)\right\rangle \simeq 8 \pi k_{B} T R t /\left(\hbar R_{K}\right),
$$

such as in a purely dissipative environment. ${ }^{22}$ At $t \sim \delta \omega / \omega_{L C}^{2}$, the phase fluctuations level off at the value given by the long-time, high-temperature limit in Eq. (11),

$$
\left\langle\phi^{2}(t)\right\rangle \simeq 8 \pi k_{B} T L_{J} /\left(\hbar R_{K}\right) .
$$

In the limit $\delta \omega / \omega_{L C} \gg 1$, the circuit attains features, on one hand, of an inductance limited phase diffusion measurement and, on the other hand, of a dissipatively dephasing environment.

Illustrations of the results of this section for model circuit $\mathbf{B}$ are shown in Fig. 5. In Fig. 5(a), we see the results of numerically calculated $\left\langle\phi^{2}(t)\right\rangle$ for typical parameter values and Fig. 5(b) shows $\operatorname{Re} Z(\omega)$ with the same parameter val- 
ues. Figure 5(c) demonstrates the crossover suggested by Eqs. (12) and (13) in the dissipative case; the corresponding $\operatorname{Re} Z(\omega)$ is shown in Fig. 5(d).

The analysis of the mean square of the fluctuating phase performed in this section indicates that the measuring circuits $\mathbf{A}$ and $\mathbf{B}$, unlike a purely resistive environment, will not lead to a complete suppression of coherence of the CPP: the phase diffusion is essentially limited by the inductance. In the following section, we will analyze the influence of these fluctuations on the amount of (coherently) transferred charge through the pump. As we will see, fluctuations renormalize not only parameters of the pump, but may also induce (coherent) higher-order charge transfer, such as cotunneling. Both effects lead to additional corrections to the pumped charge.

\section{INFLUENCE OF THE MEASURING CIRCUIT ON THE TRANSFERRED CHARGE}

A convenient way to discuss the influence of the measuring apparatus on the charge transferred during one pumping cycle is to formulate the problem in the framework of the so-called effective action approach. This approach enables one to obtain the partition function of the pump together with its measuring environment as a path integral. For the case of an inductive environment, the degrees of freedom of the environment can be integrated out approximately, and we obtain an effective action that describes the low-energy behavior of the pump, in the presence of the measuring circuit. From this effective action, we will obtain an effective Hamiltonian for the pump, which is essentially Hamiltonian (1) up to two modifications: (i) the Josephson energy $E_{J}$ in Eq. (3) should be replaced by a renormalized value $E_{J, \text { eff }}$ and (ii) a term $\hat{H}_{\text {ind }}$ should be added, which accounts for correlated tunneling events induced by the measuring circuit. Both modifications will lead to corrections to result (6).

\section{A. Effective action}

We start our analysis by considering the total equilibrium partition function of the pump together with the measuring circuit,

$$
Z_{\text {tot }}=\int \mathcal{D} \phi_{1} \mathcal{D} \phi_{2} \mathcal{D} \phi e^{-S}
$$

where the Euclidean action $S$ of the system is given by $S$ $=S_{C}+S_{J}+S_{\text {bath }}$. Here, $S_{C}$ and $S_{J}$ are the actions associated with the Hamiltonians $\hat{H}_{C}$ and $\hat{H}_{J}$ defined in Eqs. (2) and (3), respectively. Specifically, the action $S_{J}$ is given by

$$
S_{J}=E_{J} \sum_{i=1}^{3} \int_{0}^{\beta} d \tau \cos \left[\delta \phi(\tau)_{i}+\phi(\tau) / 3\right],
$$

where we introduced the phase differences across each junction in the CPP: $\delta \phi_{1}=\varphi_{0} / 3+\phi_{1}, \delta \phi_{2}=\varphi_{0} / 3+\phi_{2}-\phi_{1}$, and $\delta \phi_{3}=\varphi_{0} / 3-\phi_{2}$. The third term, $S_{\text {bath }}$, is the action describing the measuring circuit. Due to this contribution, the phase bias $\varphi$ has acquired a dynamical part $\phi(\tau)$, such that $\varphi(\tau)$ $=\varphi_{0}+\phi(\tau)$. The action $S_{\text {bath }}$ can be written as

$$
S_{b a t h}=\frac{1}{2} \int_{0}^{\beta} \int_{0}^{\beta} d \tau d \tau^{\prime} \phi(\tau) D_{\phi}\left(\tau-\tau^{\prime}\right) \phi\left(\tau^{\prime}\right),
$$

where $\beta=1 / T$ (in this section, we use units such that $\hbar$ $\left.=k_{B}=1\right)$. The kernel $D_{\phi}(\tau)$ is related to the impedance of the measuring circuit, ${ }^{23} D_{\phi}(\tau)=T \Sigma_{n} D_{\phi}\left(i \omega_{n}\right) e^{-i \omega_{n} \tau}$, where

$$
D_{\phi}\left(i \omega_{n}\right)=\frac{1}{4 e^{2}} \frac{\left|\omega_{n}\right|}{Z\left(i\left|\omega_{n}\right|\right)} .
$$

Here, $\omega_{n}$ is a bosonic Matsubara frequency, $\omega_{n}=2 \pi n T$. It is possible to get an effective action depending on the phases $\phi_{1}(\tau)$ and $\phi_{2}(\tau)$ of the islands only. We write the total partition function as

$$
Z_{\text {tot }} \simeq \int \mathcal{D} \phi_{1} \mathcal{D} \phi_{2} e^{-S_{\text {eff }}} .
$$

To second order in $E_{J}, S_{\text {eff }}$ is found by a reexponentiation of the averages $\left\langle S_{J}\right\rangle$ and $\left\langle S_{J}^{2}\right\rangle$ over the action $S_{b a t h}$. For the average of $S_{J}$, we find

$$
\left\langle S_{J}\right\rangle=E_{J, \mathrm{eff}} \sum_{i=1}^{3} \int_{0}^{\beta} d \tau \cos \delta \phi_{i}(\tau),
$$

where we introduced the renormalized Josephson coupling

$$
E_{J, \mathrm{eff}}=E_{J} e^{-G_{\phi}(\tau=0) / 18},
$$

with the phase-phase correlation function

$$
G_{\phi}(\tau)=T \sum_{n} D_{\phi}^{-1}\left(\omega_{n}\right) e^{-i \omega_{n} \tau} .
$$

For the average of $S_{J}^{2}$, we obtain

$$
\begin{aligned}
\left\langle S_{J}^{2}\right\rangle= & \frac{E_{J, \mathrm{eff}}^{2}}{2} \sum_{i, j=1}^{3} \int_{0}^{\beta} d \tau_{1} d \tau_{2} \\
& \times\left\{\cos \delta \phi_{i}\left(\tau_{1}\right) \cos \delta \phi_{j}\left(\tau_{2}\right) \sum_{\sigma= \pm 1} e^{\sigma G_{\phi}\left(\tau_{1}-\tau_{2}\right) / 9}\right. \\
& \left.+\sin \delta \phi_{i}\left(\tau_{1}\right) \sin \delta \phi_{j}\left(\tau_{2}\right) \sum_{\sigma= \pm 1} \sigma e^{\delta G_{\phi}\left(\tau_{1}-\tau_{2}\right) / 9}\right\} .
\end{aligned}
$$

We see that at this order an interaction term appears, whose kernel depends on the correlation function $G_{\phi}(\tau)$. As already discussed in the preceding section, fluctuations in the bias phase are bound for the particular measuring environments we are considering. In the limit of small phase fluctuations, the correlations $G_{\phi} \sim\langle\phi(\tau) \phi\rangle$ remain small, allowing us to expand the exponentials in Eq. (19) with respect to $G_{\phi}$. Therefore, we can write

$$
\begin{aligned}
\left\langle S_{J}^{2}\right\rangle= & E_{J, \text { eff }}^{2} \sum_{i, j=1}^{3} \int_{0}^{\beta} d \tau_{1} d \tau_{2}\left[\cos \delta \phi_{i}\left(\tau_{1}\right) \cos \delta \phi_{j}\left(\tau_{2}\right)\right. \\
& \left.+\frac{1}{9} \sin \delta \phi_{i}\left(\tau_{1}\right) G_{\phi}\left(\tau_{1}-\tau_{2}\right) \sin \delta \phi_{j}\left(\tau_{2}\right)\right] .
\end{aligned}
$$



$S_{\text {eff }}$,

We are now in a position to obtain the effective action

$$
S_{\text {eff }}=S_{C}+S_{J, \text { eff }}+S_{\text {ind }},
$$

where

$$
S_{J, \mathrm{eff}}=E_{J, \mathrm{eff}} \sum_{i=1}^{3} \int_{0}^{\beta} d \tau \cos \delta \phi_{i}(\tau)
$$

and

$$
\begin{aligned}
S_{\text {ind }}= & -\frac{E_{J, \text { eff }}^{2}}{9} \sum_{i, j=1}^{3} \int_{0}^{\beta} d \tau_{1} d \tau_{2} \sin \delta \phi_{i}\left(\tau_{1}\right) \\
& \times G_{\phi}\left(\tau_{1}-\tau_{2}\right) \sin \delta \phi_{j}\left(\tau_{2}\right) .
\end{aligned}
$$

The influence of the measuring circuit is indeed twofold: (i) In the effective action, the bare Josephson coupling energy $E_{J}$ has been replaced by an effective, renormalized value $E_{J \text {,eff }}$; (ii) The measuring circuit correlates tunneling events at different junctions, as is seen from Eq. (22). Physically, a tunneling event occurring at time $\tau_{1}$ in junction $i$ virtually excites the measuring environment, which, upon relaxation, causes a second tunneling event in junction $j$ at time $\tau_{2}$. In other words, the environment induces higher-order tunneling events in the pump of the cotunneling type. Clearly, both (i) and (ii) will modify the charge transferred through the pump, as we will discuss in more detail below.

\section{B. Renormalized Josephson coupling}

In order to evaluate the renormalized Josephson coupling energy $E_{J, \text { eff }}$ as given by Eq. (17), we need to calculate the correlation function $G_{\phi}$, Eq. (18), at time $\tau=0$. This implies calculating the sum over all Matsubara frequencies of the quantity $Z\left(i\left|\omega_{n}\right|\right) /\left|\omega_{n}\right|$, which involves the total, imaginarytime response of the circuit, $Z\left(i\left|\omega_{n}\right|\right)$. For the circuits $\mathbf{A}$ and $\mathbf{B}$ of interest here we find

$$
\begin{aligned}
& Z_{A}(i|\omega|) /|\omega| \\
& \quad=\frac{R L+(1-\gamma) L_{s} L|\omega|}{R\left(1+L C_{p}|\omega|^{2}\right)+L_{s}|\omega|\left[1+(1-\gamma) L C_{p}|\omega|^{2}\right]}
\end{aligned}
$$

and

$$
Z_{B}(i|\omega|) /|\omega|=\frac{R L_{J}}{R\left(1+L_{J} C_{J}^{*}|\omega|^{2}\right)+|\omega| L_{J}},
$$

respectively. Note that, upon analytic continuation $i \omega_{n} \rightarrow \omega$ $+i 0$, the real part of $Z_{A, B}\left(i\left|\omega_{n}\right|\right)$ coincides with results (8) and (10), respectively.

From a closer inspection of Eqs. (23) and (24), we see that we can obtain $Z_{B}$ from $Z_{A}$ upon taking the limit $\gamma \rightarrow 1$ in Eq. (23), thereby replacing $L$ and $L_{s}$ by $L_{J}$. We will, therefore, focus on circuit $\mathbf{A}$ in what follows; the results obtained can be used to analyze circuit $\mathbf{B}$ after taking proper limits.

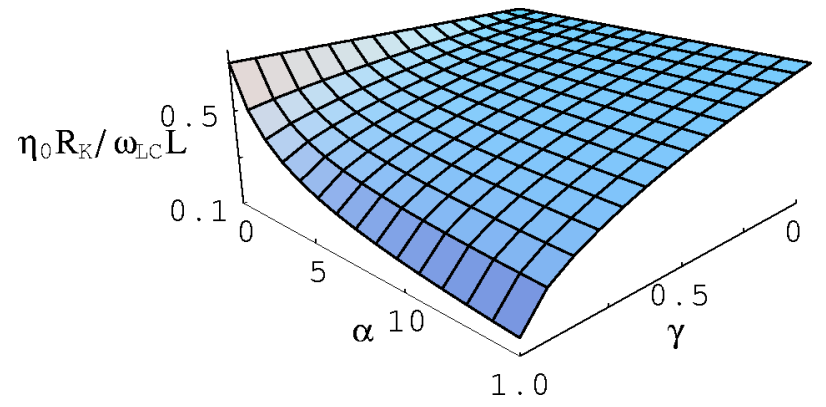

FIG. 6. Renormalization parameter $\eta_{0}$ for circuit $\mathbf{A}$ in units $\omega_{L C} L / R_{K}$ as a function of $\alpha$ and $\gamma$.

In the zero-temperature limit $T \rightarrow 0$, the sum over $\omega_{n}$ in Eq. (18) can be replaced by an integral. In this limit, the renormalized Josephson coupling energy can be written as $E_{J, \mathrm{eff}}=E_{J} e^{-\eta_{0}}$, where

$$
\eta_{0}=\frac{4 \omega_{L C} L}{9 R_{K}} \int_{0}^{\infty} d x \frac{1+\alpha(1-\gamma) x}{1+x^{2}+\alpha x\left[1+(1-\gamma) x^{2}\right]} .
$$

We introduced the dimensionless integration variable $x$ $=\omega / \omega_{L C}$ and used the parameter $\alpha=\omega_{L C} L_{s} / R$ introduced in Sec. III. Note that the integral is always finite: the integrand is well behaved, both for small values of $x$, reflecting the fact that low-frequency phase fluctuations are limited by the presence of an inductance in the circuit, and for large values of $x$, reflecting the natural high-frequency cutoff of the fluctuations provided by the presence of capacitances. In Fig. 6, we plotted $\eta_{0}$ as a function of the dimensionless quantities $\alpha$ and $\gamma$. Let us use some realistic parameter values to estimate the effect. For circuit $\mathbf{A}, \alpha \sim 1$ and $\gamma \ll 1$; hence, we have $\eta_{0} \leq 0.1$ and $E_{J, \text { eff }} \geq 0.9 E_{J}$. For circuit $\mathbf{B}, \alpha \sim 10^{-2}$ and $\gamma$ $\equiv 1$; hence, we have $\eta_{0} \lesssim 10^{-3}$ and $E_{J \text {,eff }} \simeq E_{J}$. We conclude that the renormalization of $E_{J}$ is always weak. This result confirms the analysis of Sec. III: for small values of $\alpha$ or $\gamma$ the parameter $\eta_{0}$ indeed equals the asymptotic values for $\left\langle\phi^{2}\right\rangle / 18$, see Eqs. (9) and (11). Thus, for suitably chosen parameters, the measuring circuit $\mathbf{A}$ (as well as $\mathbf{B}$ ) will not suppress the coherent coupling between various parts of the pump.

The results presented so far are valid at zero temperature, $T=0$, and in the absence of a gate modulation, $f=0$. Finite temperature introduces a correction to $\eta_{0}$ due to the summation over Matsubara frequencies in Eq. (17). As long as the inductance $L$ of the pump is small compared to the inductance $\hbar /\left(2 e I_{J}\right)$ of the Josephson junctions, this correction can be neglected at temperatures of interest here $k_{B} T \ll E_{J}$. The effect of a finite frequency $f$ can also be ignored at this point, since we are working in the leading order in the adiabatic approximation.

The above results, obtained for an inductive environment, should be contrasted with the effect of a purely resistive measuring circuit, characterized by a resistance $R_{0}$. In this case, the low-frequency impedance is constant, $Z \simeq R_{0}$, and the sum over Matsubara frequencies in Eq. (18) diverges logarithmically. This reflects the fact that the low-frequency fluctuations of the phase are unbound. As a result, the Jo- 
sephson coupling will be significantly renormalized. Indeed, in the case of a very resistive environment, characterized by a large dimensionless resistance ratio $4 R_{0} / 9 R_{K} \gg 1$, the renormalized Josephson coupling at $T=0$ is given by $E_{J \text {,eff }}$ $\sim E_{J}\left(f R_{0} C_{p}\right)^{4 R_{0} / 9 R_{K}}$, which depends explicitly on the operating frequency $f$ of the pump. This means that the Josephson energy is completely suppressed in the absence of a gate modulation, $f=0$. For finite values of $f$, the pump will be characterized by a finite Josephson coupling energy; however, its value will be small, $E_{J \text {,eff }}^{2} / E_{C} \ll \hbar f$, for any reasonable operating frequency. We thus conclude that it is practically impossible to operate the pump in the adiabatic regime if $R_{0}$ is large. In the opposite limit of a weakly resistive environment, $4 R_{0} / 9 R_{K} \ll 1$, one finds $E_{J \text {,eff }}$ $\sim E_{J}\left(E_{J} R_{0} C_{p} / \hbar\right)^{4 R_{0} / 9 R_{K}} \lesssim E_{J}$ : the renormalization of the Josephson coupling is limited. We conclude that, at least, in principle, the device can be operated coherently in the adiabatic regime in the presence of weak dissipation, as long as $R_{0}$ is sufficiently small.

\section{Calculation of the transferred charge}

We finally turn to the actual calculation of the charge transferred per cycle. In order to use Eq. (4), which involves instantaneous eigenstates of a Hamiltonian, it is convenient to transform the effective action $S_{\text {eff }}$ into an effective Hamiltonian, $\hat{H}_{\text {eff }}=\hat{H}_{C}+\hat{H}_{J \text {, eff }}+\hat{H}_{\text {ind }}$. With the help of Eq. (21), we see that $\hat{H}_{J \text {,eff }}$ can be obtained directly from Eq. (3), replacing $E_{J}$ by the renormalized value $E_{J, \text { eff }}$. The Hamiltonian $\hat{H}_{\text {ind }}$ is obtained from $S_{\text {ind }}$, which depends on the correlation function $G_{\phi}$. The fact that $G_{\phi}$ is, in general, nonlocal in time complicates matters. However, for the measuring circuit $\mathbf{A}$, the dynamics at low frequencies is dominated by the inductance $L$ (for circuit $\mathbf{B}$, it is $L_{J}$ ). Provided that $L$ is small, such that $L \ll \hbar /\left(2 e I_{J}\right)$, the relevant correlations are local in time and $G_{\phi}(\tau) \simeq\left(8 \pi L / R_{K}\right) \delta(\tau)$. In other words, the two correlated tunneling events induced by the measuring environment are instantaneous on the slow time scale characterizing the junction dynamics. Substituting the above local form for the correlator $G_{\phi}$ into Eq. (22) and performing one of the integrations over imaginary time, we see that the resulting $S_{\text {ind }}$ can be transformed into

$$
\hat{H}_{\mathrm{ind}}=-\frac{E_{J, \mathrm{eff}}}{18} \frac{E_{J, \mathrm{eff}} L}{(\hbar / 2 e)^{2}} \sum_{i, j=1}^{3} \sin \delta \phi_{i} \sin \delta \phi_{j}
$$

The calculation of $Q_{P}$ with Eq. (4) with the Hamiltonian $\hat{H}_{\text {eff }}$ for a triangular gating sequence is straightforward. Ignoring $\hat{H}_{\text {ind }}$, one obtains result (6), with $E_{J}$ replaced by its renormalized value $E_{J \text {,eff }}$. If $\hat{H}_{\text {ind }}$ is taken into account, a correction is found due to coherent higher-order tunneling events induced by the inductive environment. Referring the reader to Appendix for details, we only present the final result:

$$
Q_{P} / 2 e \simeq 1-\left(9 \frac{E_{J, \mathrm{eff}}}{E_{C}}-\frac{1}{6} \frac{E_{J, \mathrm{eff}} L}{(\hbar / 2 e)^{2}}\right) \cos \varphi_{0} .
$$

Taking $E_{J} / E_{C}=0.1$ with $E_{C} \sim 1 \mathrm{~K}$, the renormalization of $E_{J}$ is negligible. With $L=100 \mathrm{nH}$ (circuit $\mathbf{A}$ ), the second correction $E_{J, \text { eff }} L /\left[6(\hbar / 2 e)^{2}\right] \simeq 0.2$, whereas for circuit $\mathbf{B}$ this would be $E_{J, \text { eff }} L /\left[6(\hbar / 2 e)^{2}\right] \simeq 2 \times 10^{-4}$.

\section{DISCUSSION}

The CPP has a few properties that make it an attractive object of investigation both theoretically and experimentally. The mechanism for deviations of the pumped charge from the quantized value are interesting in their own right. These include Landau-Zener (LZ) band crossing, quasiparticle current, and last but not least the topic of the current paper, the coherent quantum interference in Cooper-pair transport. They all can be avoided at least, in principle, by using a favorable operation mode: LZ crossing can be minimized by operating the pump at low enough frequency, $f$ $\ll E_{J}^{2} /\left(\hbar E_{C}\right)$, quasiparticle current by low temperature and careful filtering of the sample, and coherence induced corrections by dissipative environment of the CPP.

Quantized charge transport is being investigated largely because it may eventually fulfill the requirements of providing a modern standard for electrical current. At the same time, it would close the metrological triangle of electrical quantities: by now both the voltage and resistance have their quantum standards, Josephson voltage and quantum Hall resistance, respectively, and by determining the current using $I=q f$ would test the validity of Ohm's law with the same value of Planck's constant in the two. But there are serious problems to be solved before $I$ can be determined at sufficient absolute accuracy, error rate being smaller than $10^{-7}$ and output current on the nanoamperes scale. The foremost problems are either the very small current obtained in the tunnel junction based pumps or the errors in the number of electrons carried in the moving quantum dots in the semiconductor pumps.

Cooper-pair pump may solve these problems eventually. The current limitation can perhaps be lifted by using higher values of $E_{J}$ employing Josephson junctions made of a superconductor with higher $T_{c}$ than that of aluminum $(1 \mathrm{~K})$. An obvious candidate is niobium, where $T_{c} \simeq 99$, whereby yielding an almost one order of magnitude enhancement in $E_{J} \propto T_{c}$. Fabricating small junctions using $\mathrm{Nb}$ has, however, turned out to be a challenge, which has not been fully solved yet. ${ }^{24,25}$ The second problem is how to suppress errors due to the quantum interference in the CPP without disturbing the operation of the pump otherwise. As an example, by inserting a highly dissipative termination to the pump, heating becomes a problem especially at the desired higher throughput currents. Therefore, understanding the nature of this interference is of importance in optimizing the operation of the CPP. One alternative to dissipative environment is to employ pumps with larger number of junctions, $N$, since the interference correction is proportional to $\left(E_{J} / E_{C}\right)^{N-2}$.

\section{ACKNOWLEDGMENTS}

We thank O. Buisson, G. Falci, P. Hakonen, T. Heikkilä, J. Kivioja, Ph. Lafarge, L. Lévy, A. Niskanen, M. Paalanen, H. 
Seppä, and J. Toppari for useful discussions. F.W.J.H. acknowledges support from Institut Universitaire de France. J.P.P. thanks the Center de Recherches sur les Très Basses Températures, where part of this work was done, for its hospitality. We acknowledge EC for financial support through Grant Nos. IST-FET-SQUBIT and HPRN-CT-2002-00144.

\section{APPENDIX A: DERIVATION OF Eq. (4)}

The Hamiltonian $\hat{H}$, Eq. (1), depends explicitly on the gate charges $n_{x 1}$ and $n_{x 2}$ through the charging term $\hat{H}_{C}$, Eq. (2). As a result of the periodic modulation of the vector $\vec{n}_{x}$ $=\left(n_{x 1}, n_{x 2}\right)$ as a function of time, the Hamiltonian becomes time dependent. If the modulation is adiabatically slow, Schrödinger's equation $i \hbar \partial\left|\psi_{m}(t)\right\rangle / \partial t=\hat{H}(t)\left|\psi_{m}(t)\right\rangle$ is satisfied by an adiabatic state of the form

$$
\left|\psi_{m}(t)\right\rangle=\exp \left[-i \int^{t} d t^{\prime} E_{m}\left(t^{\prime}\right) / \hbar\right] e^{i \gamma_{m}(t)}|m(t)\rangle
$$

where $|m(t)\rangle$ is an instantaneous eigenstate of $\hat{H}(t)$ with energy $E_{m}(t)$ and $\gamma_{m}$ is Berry's phase. ${ }^{26}$

The expectation value of any time-independent Hermitian operator $\hat{O}$ in the state $\left|\psi_{m}(t)\right\rangle$ is, in general, time dependent and given by $\langle O(t)\rangle=\left\langle\psi_{m}(t)|\hat{O}| \psi_{m}(t)\right\rangle=\langle m(t)|\hat{O}| m(t)\rangle$. Specifically, we see that the dynamics of $\langle O(t)\rangle$ is governed by the instantaneous eigenstates. Using the fact that $|\dot{m}\rangle$ $=\vec{n}_{x} \cdot \partial_{n_{x}}|m\rangle$, where $\dot{a} \equiv d a / d t$, we obtain

$$
\langle\dot{O}\rangle=2 \operatorname{Re} \sum_{n \neq m}\langle m|\hat{O}| n\rangle\left\langle n \mid \partial_{\vec{n}_{x}} m\right\rangle \cdot \dot{\vec{n}}_{x} .
$$

Note that the term $n=m$ can be excluded from the sum in Eq. (A1), as $\left\langle n \mid \partial_{n_{x}} n\right\rangle$ is purely imaginary. This can be seen using the normalization condition $\langle n \mid n\rangle=1$ from which we obtain $\partial_{\vec{n}_{x}}\langle n \mid n\rangle=0=2 \operatorname{Re}\left\langle n \mid \partial_{n_{x}} n\right\rangle$.

We are interested in the total charge $Q_{P}$ transferred through the pump during one cycle. Since the modulation is periodic, $Q_{P}$ is the total charge transferred through any of the junctions during the cycle, e.g., the leftmost one $Q_{l}$. Let us consider the matrix elements of the current passing through the left junction, $\left\langle m\left|\hat{I}_{l}\right| n\right\rangle$, see Eq. (5),

$$
\left\langle m\left|\hat{I}_{l}\right| n\right\rangle \equiv \frac{i}{\hbar}\left\langle m\left|\left[\hat{H}, \hat{Q}_{l}\right]\right| n\right\rangle=\frac{i}{\hbar}\left(E_{m}-E_{n}\right)\left\langle m\left|\hat{Q}_{l}\right| n\right\rangle .
$$

Applying result (A1) to the charge operator $\hat{Q}_{l}$ and using (A2), we obtain

$$
\left\langle\dot{Q}_{l}\right\rangle=2 \hbar \operatorname{Im} \sum_{n \neq m} \frac{\left\langle m\left|\hat{I}_{l}\right| n\right\rangle}{E_{m}-E_{n}}\left\langle n \mid \partial_{\vec{n}_{x}} m\right\rangle \cdot \dot{\vec{n}}_{x}
$$

Therefore, the total charge passing through the pump in the state $\left|\psi_{m}\right\rangle$ is given by

$$
Q_{P}=\int_{\text {cycle }} d t\left\langle\dot{Q}_{l}\right\rangle=2 \hbar \operatorname{Im}\left[\sum_{n \neq m} \oint \frac{\left(\hat{I}_{l}\right)_{m n}}{E_{m}-E_{n}}\left\langle n \mid \partial_{n_{x}} m\right\rangle \cdot d \vec{n}_{x}\right]
$$

which is the result, Eq. (4), upon setting $|m\rangle=|0\rangle$.

\section{APPENDIX B: TRANSFERRED CHARGE FOR TRIANGULAR GATING}

In this appendix, we outline the perturbative calculation of $Q_{P}$ with Eq. (4), for a triangular gating sequence as in Fig. 2 . This sequence corresponds to a contour consisting of three linear segments, (1), (2), and (3), in which, respectively, the gate-charge vector $\vec{n}_{x}$ is changed adiabatically from $(0,0)$ to $(1,0)$, increasing $n_{x 1}$; then from $(1,0)$ to $(0,1)$, simultaneously decreasing $n_{x 1}$ and increasing $n_{x 2}$; finally from $(1,0)$ to $(0,0)$, decreasing $n_{x 2}$. In this appendix, when evaluating $Q_{P}$, we will present explicit calculations for segment $(1)$ from $(0,0)$ to $(1,0)$ only, i.e., we will calculate

$$
Q_{P}^{(1)}=2 \hbar \operatorname{Im}\left[\sum_{n \neq 0} \int_{0}^{1} \frac{\left(\hat{I}_{l}\right)_{0 n}}{E_{0}-E_{n}}\left\langle n \mid \partial_{n_{x 1}} 0\right\rangle d n_{x 1}\right] .
$$

Results for segments (2) and (3) will be simply stated, their calculation being essentially analogous to the one for segment (1).

Since $E_{J} \ll E_{C}$, only a limited number of charge states need to be taken into account in the calculation. This enables one to proceed perturbatively; below we will consider various contributions to $Q_{P}$ that arise in different orders of perturbation theory.

\section{Incoherent contribution}

In leading order, the only relevant charge states for segment (1) of interest, here are the eigenstates $|0,0\rangle$ and $|1,0\rangle$ of $\hat{H}_{C}$, Eq. (2), which are mixed coherently by $\hat{H}_{J}$, Eq. (3). This is analogous to the coherent mixing of charge states in a single Cooper-pair box, see Ref. 15. The two lowest instantaneous eigenstates are therefore

$$
\begin{aligned}
& |0\rangle=a|0,0\rangle+e^{i \varphi_{0} / 3} b|1,0\rangle, \\
& |1\rangle=b|0,0\rangle-e^{i \varphi_{0} / 3} a|1,0\rangle,
\end{aligned}
$$

with an energy difference $E_{1}-E_{0}=E_{J} \sqrt{1+\epsilon^{2}}$. Here, $a^{2}=1-b^{2}=(1 / 2)\left(1+\epsilon / \sqrt{1+\epsilon^{2}}\right) \quad$ such that $a b$ $=1 /\left(2 \sqrt{1+\epsilon^{2}}\right)$. The dependence on the parameter $n_{x 1}$ enters through $\epsilon=\left(2 E_{C} / 3 E_{J}\right)\left(1-2 n_{x 1}\right)$. Higher states can be ignored as they are separated in energy by an amount $\sim E_{C}$.

We proceed by evaluating the various terms appearing in Eq. (B1). In order to obtain the matrix elements $\left(\hat{I}_{l}\right)_{01}$ for current through the leftmost junction, it is convenient to use the charge representation of Eq. (5). Putting $\varphi=\varphi_{0}$, we find 


$$
\begin{aligned}
\hat{I}_{l}= & \frac{I_{J}}{2 i} \sum_{n_{1}, n_{2}}\left(e^{i \varphi_{0} / 3}\left|n_{1}, n_{2}\right\rangle\left\langle n_{1}+1, n_{2}\right|\right. \\
& \left.-e^{-i \varphi_{0} / 3}\left|n_{1}+1, n_{2}\right\rangle\left\langle n_{1}, n_{2}\right|\right) .
\end{aligned}
$$

A direct calculation, using the above decomposition of $|0\rangle$ and $|1\rangle$ into charge states, then yields $\left(\hat{I}_{l}\right)_{01}=-I_{J} / 2 i$. Similarly, the decomposition can be used to calculate $\left\langle 1 \mid \partial_{n_{x 1}} 0\right\rangle$ $=b \partial_{n_{x 1}} a-a \partial_{n_{x 1}} b$. Finally, using the equality $E_{1}-E_{0}$ $=E_{J} / 2 a b$, we obtain, in leading order,

$$
\begin{aligned}
Q_{P}^{(1)} & \simeq 4 e \int_{0}^{1} a b\left(a \partial_{n_{x 1}} b-b \partial_{n_{x 1}} a\right) d n_{x 1} \\
& \simeq 8 e \int_{0}^{1} a\left(1-a^{2}\right) d a=2 e .
\end{aligned}
$$

In this order, the contributions from segments (2) and (3) are zero, and we conclude that $Q_{P}=2 e$. Indeed, in the absence of higher-order tunneling processes, the current through the leftmost junction is not affected by the coherent mixing of charge states $|1,0\rangle$ and $|0,1\rangle$ along segment (2) or of $|0,1\rangle$ and $|0,0\rangle$ along segment (3). Below, we will include higher-order tunneling which leads to corrections to these results of the order of $E_{J} / E_{C}$.

\section{Coherent correction}

We now take into account the charge states that $\hat{H}_{J}$ mixes into $|0\rangle$ and $|1\rangle$ by second-order tunneling processes. For segment (1) of interest, these are the charge states $|0,1\rangle$ and $|1,-1\rangle$. Straightforward second-order perturbation theory yields the correction to the states $|0\rangle$ and $|1\rangle$,

$$
\delta|0\rangle=c|1\rangle \text { and } \delta|1\rangle=c^{*}|0\rangle
$$

where

$$
c=\left(3 E_{J} / E_{C}\right) a b\left(b^{2} e^{i \varphi_{0}}-a^{2} e^{-i \varphi_{0}}\right) .
$$

The energies $E_{0}$ and $E_{1}$ are also renormalized, yielding a correction to the energy difference

$$
\delta\left(E_{1}-E_{0}\right)=\left(6 E_{J}^{2} / E_{C}\right) a b \cos \varphi_{0} .
$$

Note the appearance in this order of a dependence of the corrections on the bias phase $\varphi_{0}$. Along segment (1), phase coherence through the entire pump is established by coherent mixing at the leftmost junction in combination with secondorder tunneling through the middle and rightmost junctions.

The matrix elements of $\hat{I}_{l}$ are not affected by the corrections. Using Eq. (B6), we obtain $\delta\left(\hat{I}_{l}\right)_{01}=c^{*}\left[\left(\hat{I}_{l}\right)_{00}\right.$ $\left.+\left(\hat{I}_{l}\right)_{11}\right]$, which vanishes since the instantaneous eigenstates carry no current: $\left(\hat{I}_{l}\right)_{00}=\left(\hat{I}_{l}\right)_{11}=0$, as can be seen easily from Eqs. (B2)-(B4). However, $\delta\left\langle 1 \mid \partial_{n_{x 1}} 0\right\rangle$ is nonvanishing, $\delta\left\langle 1 \mid \partial_{n_{x 1}} 0\right\rangle=\partial_{n_{x 1}} c$. The resulting correction to $Q_{P}^{(1)}$ can be written as the integral

$$
\begin{aligned}
\frac{\delta Q_{P}^{(1)}}{2 e}= & \frac{E_{J}}{E_{C}} \cos \varphi_{0} \int_{0}^{1} d n_{x 1}\left\{24(a b)^{3}\left(b \partial_{n_{x 1}} a-a \partial_{n_{x 1}} b\right)\right. \\
& \left.-6 a b\left[\left(b^{3}-3 a^{2} b\right) \partial_{n_{x 1}} a-\left(a^{3}-3^{2} a\right) \partial_{n_{x 1}} b\right]\right\},
\end{aligned}
$$

where the first term stems from the energy renormalization (B8) and the second term from the correction $\delta\left\langle 1 \mid \partial_{n_{x 1}} 0\right\rangle$, calculated taking a derivative in Eq. (B7). Direct integration yields

$$
\delta Q_{P}^{(1)} / 2 e=-3 \frac{E_{J}}{E_{C}} \cos \varphi_{0} .
$$

A similar calculation for segments (2) and (3) yields the same result. In other words, the total correction to $Q_{P}$ is given by $\delta Q_{P}=-2 e\left(9 E_{J} / E_{C}\right) \cos \varphi_{0}$, in agreement with Eq. (4).

\section{Correction due to inductive coupling}

We finally consider the corrections to $Q_{P}$ associated with the Hamiltonian $\hat{H}_{\text {ind }}$, Eq. (25), which depends quadratically on $E_{J}$. Hence, first-order perturbation theory in $\hat{H}_{\text {ind }}$ yields a correction to $Q_{P}$ which is linear in $E_{J}$. We first find the corrections to the lowest instantaneous eigenstates

$$
\delta|0\rangle=d|1\rangle \text { and } \delta|1\rangle=-d^{*}|1\rangle
$$

where

$$
d=\left\{E_{J, \mathrm{eff}} L /\left[18(\hbar / 2 e)^{2}\right]\right\} a b\left(a^{2} e^{-i \varphi_{0}}-b^{2} e^{i \varphi_{0}}\right)
$$

The energy difference is renormalized as well,

$$
\delta\left(E_{1}-E_{0}\right)=-\left\{E_{J, \mathrm{eff}}^{2} L /\left[9(\hbar / 2 e)^{2}\right]\right\} a b \cos \varphi_{0} .
$$

Comparing these results with the corresponding ones found in the preceding section, Eqs. (B6)-(B8), we conclude that the subsequent calculation will be completely equivalent to the one performed in the preceding section. Indeed, the matrix elements of $\hat{I}_{l}$ are not affected by the corrections, and the contribution from $\delta\left\langle 1 \mid \partial_{n_{x 1}} 0\right\rangle$ is equal to $\partial_{n_{x 1}} d$. As a result, the correction $\delta Q_{P \text {,ind }}^{(1)}$ can be presented as an integral similar to the one in Eq. (B9). Segments (2) and (3) give the same contribution and the total correction to $Q_{P}$ due to $\hat{H}_{\text {ind }}$ is therefore given by

$$
\delta Q_{P, \text { ind }}=2 e\left\{E_{J, \mathrm{eff}} L /\left[6(\hbar / 2 e)^{2}\right]\right\} \cos \varphi_{0},
$$

in agreement with Eq. (26). The result is perturbative, the parameter $E_{J, \text { eff }} L /(\hbar / 2 e)^{2}$ must be small compared to unity. This is in agreement with the condition $L \ll\left(\hbar / 2 e I_{J}\right)$ stated in the main text for the derivation of the Hamiltonian $\hat{H}_{\text {ind }}$. 
${ }^{1}$ D.J. Thouless, Phys. Rev. B 27, 6083 (1983).

${ }^{2}$ See, e.g., M. Moskalets and M. Büttiker, Phys. Rev. B 66, 205320 (2002), and references therein.

${ }^{3}$ P.W. Brouwer, Phys. Rev. B 58, R10 135 (1998).

${ }^{4}$ Single Charge Tunneling, edited by H. Grabert and M. H. Devoret (Plenum Press, New York, 1992).

${ }^{5}$ D.V. Averin and Yu.V. Nazarov, Phys. Rev. Lett. 65, 2446 (1990).

${ }^{6}$ M. Switkes, C.M. Marcus, K. Campman, and A.C. Gossard, Science 283, 1905 (1999).

${ }^{7}$ H. Pothier, P. Lafarge, C. Urbina, D. Esteve, and M.H. Devoret, Europhys. Lett. 17, 249 (1992).

${ }^{8}$ L.P. Kouwenhoven, A.T. Johnson, N.C. van der Vaart, C.J.P.M. Harmans, and C.T. Foxon, Phys. Rev. Lett. 67, 1626 (1991).

${ }^{9}$ L.J. Geerligs, S.M. Verbrugh, P. Hadley, J.E. Mooij, H. Pothier, P. Lafarge, C. Urbina, D. Esteve, and M.H. Devoret, Z. Phys. B: Condens. Matter 85, 349 (1991).

${ }^{10}$ J.P. Pekola, J.J. Toppari, M. Aunola, M.T. Savolainen, and D.V. Averin, Phys. Rev. B 60, 9931 (1999).

${ }^{11}$ Y. Nakamura, Yu. Pashkin, and J.S. Tsai, Nature (London) 398, 786 (1999).

${ }^{12}$ J.R. Friedman, V. Patel, W. Chen, S.K. Tolpygo, and J.E. Lukens, Nature (London) 406, 43 (2000).

${ }^{13}$ C.H. van der Wal, A.C.J. ter Haar, F.K. Wilhelm, R.N. Schouten,
C.J.P.M. Harmans, T.P. Orlando, S. Lloyd, and J.E. Mooij, Science 290, 773 (2000).

${ }^{14}$ D. Vion, A. Aassime, A. Cottet, P. Joyez, H. Pothier, C. Urbina, D. Esteve, and M.H. Devoret, Science 296, 886 (2002).

${ }^{15}$ Yu. Makhlin, G. Schön, and A. Shnirman, Rev. Mod. Phys. 73, 357 (2001).

${ }^{16}$ G.L. Ingold and Yu. V. Nazarov, in Single Charge Tunneling (Ref. 4), p. 21.

${ }^{17}$ G. Schön and A.D. Zaikin, Phys. Rep. 198, 237 (1990).

${ }^{18}$ J.M. Martinis, S. Nam, J. Aumentado, K.M. Lang, and C. Urbina (unpublished).

${ }^{19}$ M.W. Keller, J.M. Martinis, N.M. Zimmerman, and A.H. Steinbach, Appl. Phys. Lett. 69, 1804 (1996).

${ }^{20}$ S.V. Lotkhov, S.A. Bogoslovsky, A.B. Zorin, and J. Niemeyer, Appl. Phys. Lett. 78, 946 (2001).

${ }^{21}$ O. Buisson, F. Balestro, J.P. Pekola, and F.W.J. Hekking, Phys. Rev. Lett. 90, 238304 (2003).

22 J.P. Pekola and J.J. Toppari, Phys. Rev. B 64, 172509 (2001).

${ }^{23}$ A.J. Leggett, Phys. Rev. B 30, 1208 (1984).

${ }^{24}$ N. Kim, K. Hansen, J. Toppari, T. Suppula, and J. Pekola, J. Vac. Sci. Technol. A 20, 386 (2002).

${ }^{25}$ R. Dolata, H. Scherer, A.B. Zorin, and J. Niemeyer, Appl. Phys. Lett. 80, 2776 (2002).

${ }^{26}$ M.V. Berry, Proc. R. Soc. London, Ser. A 392, 45 (1984). 